\title{
The Study of Data Security in Cloud Computing
}

\author{
Abdullahi Abubakar, Alhaji Idi Babate, Abdulhakeem Ishola, and Asma'u Muhammad Sani
}

\begin{abstract}
Cloud computing holds the possibility to annihilate the needs for setting up of costly computing infrastructure for IT-based services and proffering IT Solutions. It offers an adaptable IT architecture which is accessible via the internet for lightweight and hand-held compact gadgets. The concept has encouraged numerous folds to improve in the capacity or capabilities of the existing and new software. In the cloud computing environment, the whole data/information is available over a set of networked resources, empowering the information to be accessible via virtual machines. Considering the fact that the cloud computing server may be located in any part of the world beyond the reach and control of users, there are various security issues and privacy challenges that need to be clearly understood with a view to identifying how to mitigate them. Likewise, there are possibilities of the imminent breakdown of servers which have been witnessed in recent times. This research addresses the security issues connected with cloud computing, provides analysis of the militating factors against the successful implementation of cloud computing and proffers useful recommendations on how to ameliorate identified issues.
\end{abstract}

Index Terms-Cloud Computing, Data Security, Computer Network, Virtual Machine, Privacy.

\section{INTRODUCTION}

Cloud computing is a vision that has long been conceived as a potential utility where cloud clients can, over a distance, save data into the cloud in order to benefit from services and applications stored in a remote server and process the data remotely over a network of resources [1].

Cloud computing [2] has been described as an evolving discipline in this modern era and the term has acquired a lot of footing being propagated through advertisements over Internet, providers of website service hosting, providers of cloud data service to providers of virtualization software. There has been a computing paradigm shift over the last century as illustrated in Fig. 1.

Cutting through the hype of the term "cloud computing" is not an easy task. A simple web search suffices in order to discover that there are as many definitions of what constitutes "cloud computing" as there are organizations in existing market in search of exploiting new areas in this favourable emerging commercial field. Many companies that have been known to be active in the field of providing suites of cloud computing services and these include; Microsoft Company, IBM Corporation, Amazon Company,

Published on August 31, 2020.

Abdullahi Abubakar, Waziri Umaru Federal Polytechnic, Nigeria. (e-mail: abafo22@gmail.com)

Alhaji Idi Babate, Federal College of Education (Tech.), Nigeria. (e-mail: ababate2002@ gmail.com)

Abdulhakeem Ishola, Waziri Umaru Federal Polytechnic, Nigeria. (e-mail: abduljedo@ gmail.com)

Asma'u Muhammad Sani, Kaduna Polytechnic, Nigeria.

(e-mail: asmausn@gmail.com)
Google Company, Yahoo Company and Apple Inc., among others [3]. Mckendric [4], points out that there are enterprises engaged in the provisions of cloud computing as commercial solutions in one form or the other and have been actively sponsoring researches in research centres and promoting the advancement of marketable technology. Marketing expressions, aside from the academia, are reported in [5] to have also proffered meaning and explanations of what the aims and purposes of cloud computing should be, and these include typical services like providing virtual servers, data storage facilities, etc. The services are outlined, for instance, in the works of the GRIDS laboratory at University of Melbourne and in RAD laboratory at the University of California, Berkeley where a group of scientists produced a white paper called "Above the Clouds: A Berkeley View of Cloud Computing" [5].

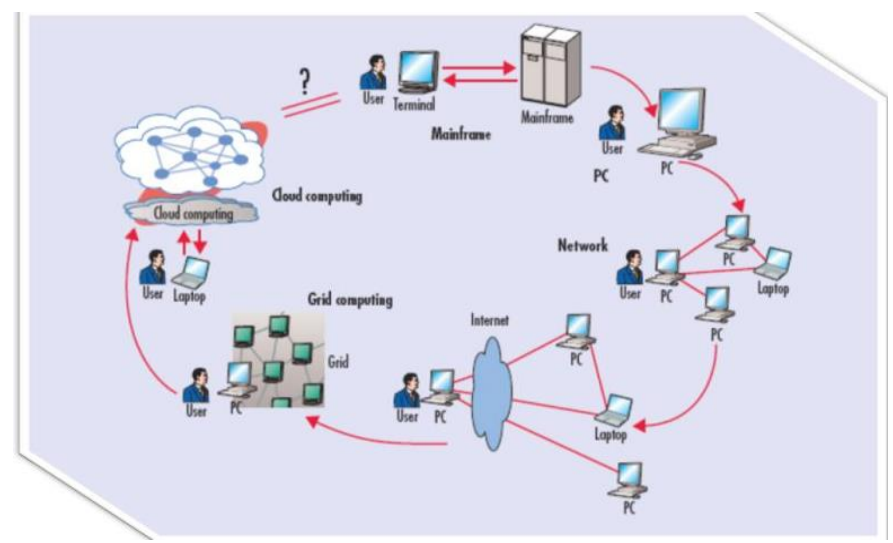

Fig. 1. History of Cloud Computing [2].

ORACLE [6], explains that many notable companies such as Oracle are eager to provide "new offerings that allow enterprises to benefit from the developments taking place in the area of Cloud Computing." However, these companies have attempted to steer out of the hype, as asserted by Oracle's Larry Ellison, by highlighting that they have "redefined cloud computing to include everything that we already do" [7].

Intel IT Centre [8], oines that in line with the popularity and interest in cloud computing, however, there are rising concerns on data security with data stored in cloud storage as results of the vulnerability of the data, unpredictable services and malicious intrusions from hackers.

Recently, more and more events on cloud service outage or server corruption with major cloud infrastructures providers are being reported. In June 2012, for example, "parts of Amazon Web Services (AWS) suffered an outage which led to a spread of high-profile sites clocking off the web for the evening" [9].

Data breaching of noteworthy cloud services also occasionally occur, as evident in the following examples:

SONY: As reported in "The six worst data breaches of 2011 " [10] points out that Sony suffered over a dozen data 
breaches, stemming from attacks that compromised the Sony PlayStation Network, Sony online entertainment and Sony pictures, among other Sony-owned websites.

EPSILON: Epsilon, a cloud-based email service provider, fell victim to a spear-phishing attack in April 2011. The breach affected data from 75 of Epsilon's clients who have entrusted Epsilon with their customers' data. Conservative estimates suggest that 60 million customer email addresses were compromised [10].

Cloud service providers may also voluntarily examine customers' data for various motivations as discussed in a report produced by [11] for Worcester Polytechnic Institute. It is argued that the Cloud is neither secure nor reliable from the viewpoint of cloud customers.

Security appears to be one of the vital issues for Cloud success. Many surveys like the one performed by the top tech executives in [12] show that security in the services available in the Cloud is now the major security challenge of cloud computing. Before a few years ago, all the business transaction processes of business organizations were on their private facilities and, though it was then possible to outsource needed services, it was often data/applications that are not critical that are on their private infrastructures

With the invention of cloud computing, the mode of processing business transactions has changed. The conventional network boundary is broken, and business organizations feel that they have lost private control over their stored data [13]. Jacob [14], explains that the new possibility of attack has emerged, and the benefit of data being accessible from anywhere has become the main threat.

United States Department of Homeland Security Statistics [15], revealed in that " 37,000 attempted breaches of government and private computer systems were reported in fiscal 2007, which ended in September, marking a dramatic increase from the 24,000 reported in 2006". Furthermore, "Cyber-attacks against federal agencies rose 152\% during the same period, from 5,143 to 12,986 which proves that cyber-attackers are trying to take advantage of the accessibility characteristics of the cloud computing and the internet in general" [15].

IDC [12] affirms that a considerable lot of the issues influencing cloud computing today are like those factors affecting services under the traditional computing environment. However, the problems are presented in a new environment with peculiar challenges, and this requires a consideration of the identified risks within the new environment by identifying critical areas in the cloud computing environment. The Cloud Security Alliance (CSA) identifies twelve (12) critical areas of concerns in the cloud computing environment. This can be broadly classified into two categories: "governance and operations" [16]. Governance and operations are critical and should be considered when considering the security of the cloud environment.

Organisations trying to reduce costs of operation might consider cloud computing as an alternative mode of operation because it offers significant reduction in costs [4]. Jacob in [14] further points out new companies seeking for a share in the market are seeking for an economic solution that promotes concentration on their businesses without consideration of how to setup and operate IT infrastructure needed for the business.

Considering the major security issues and threats associated with cloud computing, this research is an attempt to provide an understanding of the problems associated with outsourcing, processing (secured and reliable) and effective storage of the data. This will assure the availability of data and infrastructures when needed with a view to addressing the identified problems and promote the broad adoption of data outsourcing. Therefore, the objectives of the study are:

i. To identify the state of the art of security issues and challenges in the cloud computing environment.

ii. To recognize the best figuring security standards for information stockpiling by researching other existing model advisers for information security.

iii To make useful recommendation on how to make data and operations secured in the cloud settings.

\section{MethodOLOGY}

This research makes use of secondary sources of data by reviewing the previous works of scholars in the area of cloud computing. Reviews of works that are related to security issues in cloud computing found in Journal articles, Conference proceedings, White Papers and Government Gazettes were carried out. The research employs a critical literature review of works that present analyses of the state of the art of cloud computing security and studies on issues relating to the security of data in the cloud.

The research, therefore, figured out established policies that can enhance the security of data in cloud computing and other related studies on security in the cloud. It also explores content analysis to analyse the identified cloud security threats and how to deal with security challenges.

\section{LITERATURE REVIEW}

Cloud computing is a concept that is common in recent years and there have been numerous discussions on various aspects of the cloud computing. Print and non-print media have been used to discuss issues relating to cloud computing. It is, today, extensively discussed within the technology and business worlds; thus, there are multiple definitions. The National Institute of Standards and Technology (NIST) in [17] provides a popular description for cloud computing detailing out its characteristics, models governing it services and models employed for deployments. The NIST also contributed by providing a guiding document detailing out guidelines on security for cloud computing [18].

CSA in [19] has been recognized in the past few years for remarkable contributions to the discipline of Cloud computing. The guiding document on security in cloud computing has provided valuable insights into concepts of cloud computing which has supported definition by NIST. The guiding document also identified twelve (12) areas of security concerns by explaining and providing recommendations for dealing with each of them. Furthermore, CSA has been acknowledged to have contributed other documents such as "top threats to cloud computing" [20].

However, other sources have contributed to providing meanings, guiding principles and useful recommendations 
for dealing with identified threats. Notable amongst the contributors are Jericho Forum in [21], European Network and Information Security Agency in [22] and [23]. In the documents, various aspects of cloud computing are reviewed by identifying the benefits and risks associated with cloud computing and proffer useful suggestions needed to secure services in Cloud computing.

Notable authors through their works found in [13], [24] and [25] have also contributed in the areas of providing comprehensive definitions, security challenges and compliance as well as other related areas in cloud computing.

To further complement understanding of concept of cloud computing and its associated security issues, [12] and [26] further provide all-inclusive definitions and explanation of important related concepts.

\section{A. Autonomic and Utility Computing}

The foundation of cloud computing can be traced to the concepts of autonomic and utility computing, and they need to be explained to provide understandings of the purpose, goals and needed strategies of cloud computing.

\section{i) Autonomic Computing}

Murch [27], explains that Autonomic Computing Systems are systems that can, on its own, self-configure, selfrecuperating, self-upgrading and self-ensuring. Autonomic Computing can also be described as a self-managing computing model named after and designed based on the human body's autonomic sensory system [28]. Murch [27], further asserts that this model would manage the operations of computers' applications and their associated systems without cognizant input from the system user just like the autonomic nervous system self-regulates body systems without conscious input from human being. They act on policies of users and cloud services requirement to provide intelligent and dynamic operations.

Autonomic Computing Initiative was introduced by IBM in the year 2000 with the vision of developing selfmanaging systems [29]. Furthermore, Kephart in [30] states that: "With the expansion of the computer industry, with examples being a highly efficient networking hardware and powerful CPUs, autonomic computing is an evolution to cope with the rapidly growing complexity of integrating, managing and operating computing system"

Autonomic computing has been stimulated by researches in natural and socio-economic networks. The main focus is the development of elements of autonomic that can combat the ever-increasing complexity of numerous integrated and interconnected diverse software that have continually being developed exponentially across all areas of IT [27].

IBM identified four broad concepts that would differentiate traditional computing from autonomic computing:

i. Self-management - By employing high-level policies, automatic configuration of system components can be achieved. This promotes smooth and unified adjustment of the rest of the system.

ii. Self-optimization - individual components, on their own, struggle proactively to enhance their own performances. This effort would, in no doubt, promote improvement of efficiency of the entire system.

iii.Self-healing - the whole system in general diagnoses and solve software (in some cases, hardware as pointed out by IBM) issues. Therefore, the system is ideally selfrepairing and self-maintaining to some reasonable degree.

iv. Self-protection - the system offers self-defense against malicious attacks and system failures. IBM has also points out an "early warning" process to prevent failures that are systemic [30].

The four characteristics can be used to come up with desired characteristics that one would expect or desire that the systems deployed in cloud should have.

Computing systems are expected to be effective and serve the intended purpose when introduced into an organisation and continue to be useful as situations change. This has been stated in IBM whitepaper:

"The realization of autonomic computing will result in a significant improvement in system management efficiency. The disparate technologies that manage the environment work together to deliver the best performance results" [31]

IBM [31], opines that the idea of high degree of automation might appear as science fiction which is not realizable and that increasing autonomic systems, in their vision, would not just happen by accident but, rather, by gradually adding more and more sophisticated autonomic elements to existing humanly managed elements as engineers. However, it is further state in [31] that: "In addition to the two necessary attributes to autonomic computing, the context of cloud computing would seem to be problematic and irrelevant right now, long before the significant engineering challenges towards developing all the advanced autonomic systems are overcome"

\section{ii) Utility Computing}

Utility computing is the next important idea that will be stumbled upon after autonomic computing in cloud computing models. Buyya et al [32], confirms that the concept of utility computing is by no means a new concept that been pointed out in one form or the other since 1960s. Utility Computing has been described in [33]: "as a service provisioning model in which a service provider makes computing resources and infrastructure management available to the customer as needed, and charges them for specific usage rather than a flat rate"

Just like other forms of on-demand computing system, the model does seek to optimize the efficiency of the use of associated resources and minimize its related costs [28]. This also provide signal that it is natural that at a point, computing power would be provided as a service based on standard and billed on actual degree of usage with a little or no predetermined charges.

The inherent problem with this type of standardized service is, in actual sense, the terms and conditions governing Service Level Agreement (SLA).

"This is usually an agreement between the cloud computing provider that promises to deliver a certain service as a utility, such as storage and / or processing power in the cloud and the client that needs a certain level of Quality of Service that needs to be guaranteed - monitored and enforced" [34].

Importance must be attached to measures of guaranteed uptimes in Service Level Agreement. The quality of service measure of guaranteed uptime is paramount to SLA's. Uptime requirements are estimated to be currently anchored at $99.99 \%$ in the SLAs of most enterprises because there is a necessity to do so for all applications.

The word "utility" is used to provide analogy between essential services, such as electricity supply, that intends to 
meet increasingly changing customer needs, and charges for the resources based on degree of usage instead of fixed-rate basis [28]. This approach, sometime known as pay- as-youuse or metered services is very common in enterprise computing and is, sometime, employed for the consumer billing for use of resources; for internet service usage, web site access, file sharing access and other computer applications [3].

\section{B. Characteristics of Cloud Computing}

Cloud computing resources are available on-demand and can be acquired, used and relinquished by a client without any form of human interaction with the cloud service provider [35]. This automated process reduces the operating cost of the cloud provider for paying personnel reducing the prices at which the services are offered. In the following ways as pointed out in [35]:

Resource Pooling: By employing virtualization technique, cloud service provider can pool computing resources. These pooled resources enable the sharing of virtual and physical resources by various consumers, "dynamically assigning and releasing resources according to consumer demand" [17]. The clients do not have explicit knowledge of the physical location of the used resources except in situations when the clients request to limit the physical location of data as a result of legal obligation

Broad Network Access: Cloud services are available over the network through standardized interfaces. The network provides access to the cloud service not only by sophisticated devices such as personal computers, but also by non-complex hand-held devices such as smart phones.

Rapid Elasticity: This is the rapid matching of cloud computing resources that are available to the actual demand by the client. It means increasing rapidly the cloud capabilities for a service as the demand by clients is increasing and rapidly reducing the capabilities of the cloud computing resources when the corresponding demands drop down. This programmed process increase the efficiency of operation by decreasing the procurement time for new cloud computing capabilities when the need arises, while avoiding wastage of unused computing power when the demand has reduced.

Measured Service: As in utility computing. Cloud computing enables the measure of used resources. The measurements can be source of provision of efficient resource information to the cloud service provider and can be used to bill the consumer/client based on a payment model known as "pay-per-use"[37]-[38]. For instance, the bill that would be given to a customer for data transfer volumes may be calculated from the number of hours a service is running, or the volume of the data stored per month.

\section{Cloud Service Models}

Software-as-a-Service (SaaS): The model provides applications services to the consumer, by making use of standardized interfaces. The software services run over a cloud infrastructure, which is not visible to the costumer. The cloud service provider is charged with the responsibility of managing the application, operating systems and essential infrastructure for the provision of the services. The consumer only have the control of some of the user-specific configuration settings of the application [35].
Platform-as-a-Service (PaaS): The model provides the services as operation and development platforms to the consumers [35]. The consumers can use the platform to develop and run their own applications, supported by a cloud-based infrastructure [35].

The consumer has no control of the cloud service infrastructure which includes the network, network servers, supporting operating systems, or physical storage, but can exercise control over deployed applications by the provider and perhaps the configurations of application hosting environment [17].

Infrastructure-as-a-Service (IaaS): This service model is considered to be the lowest service in the hierarchy of models in the technology stack. It provides infrastructural resources as services, such as storage for raw data, processing power and network capacity. The consumers can then make use of IaaS based service provisions to use their own operating systems and applications, which offers a broad variety of deployment possibilities for clients better than what the PaaS and SaaS models offer. "The consumer does not manage or control the underlying cloud infrastructure but has control over operating systems, storage, deployed applications, and possibly limited control of select networking components (e.g., host firewalls)" [17].

The above presented services that can produce a form of stack as depicted in Fig. 2.

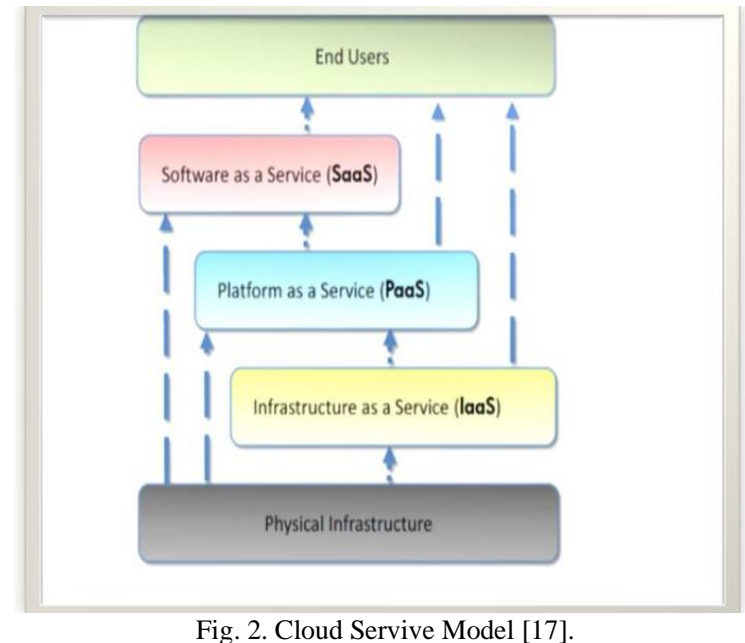

For instances, in IaaS service, consumers can deploy and implement software, with cloud service provider only managing the underlying cloud infrastructure. Also, in PaaS, consumers can deploy applications, that were created using programming languages and tools supported by CSP, onto cloud with the infrastructure being run by the CSP [35]. Furthermore, in SaaS, consumers can use CSP's applications being run on a cloud infrastructure that is provided, typically, by other CSPs [35]. In reality, IT vendors have been providing cloud services that usually include combination of elements from several layers.

There are other known forms of services provided in cloud computing and these include data as a service, business processing as a service, file security as a service, and storage as a service etc.

\section{Data Security in The Cloud}

Cloud computing as services has introduced a great number of advantages to the world of computing. In any case, security has been identified as the most important issue 
needed to be tackled in order to sustain and continuously promote the extensive use of cloud computing. Cloud computing service providers are expected to address the identified common security challenges being faced by conventional communication system [36]. Furthermore, CSPs have to look into other related issues that have been inherently introduced by the cloud computing concept itself. The security and privacy issues are the major areas of concern in cloud computing to assure that reliable and accurate data is used in a secured environment. As a result of the fact that clients can remotely log in from any location to access data and applications in the cloud, there is the possibility that the client's privacy could be compromised [36]. Therefore, Cloud service providers are expected to provide means of assuring privacy of their clients. This dictates that a prospective user of cloud services would need to review policies on data security of vendors before using their services to avoid catastrophic loss of data or losing access right to their data. A consulting firm and technology analyst, Gartner, in [23] identified seven security areas which consumers should inquire from a cloud - computing vendor before using their facilities:

1) Access Privilege - There is need to find out the level of access privilege to stored data by others and confirm the level of administrators' rights and privileges to user's data.

2) Compliance with relevant Regulations - Confirm the level of compliance of vendors with regulations governing storage and use of cloud services and ensure that the vendor has provisions for external audits and security certifications.

3) Control over Data Location - Do the providers allow control over the location of the data and to what extent?

4) Segregation of Data - Ensure that encryption facility is provided in all stages and the encryption facilities are designed, implemented and evaluated by professionals of standing experiences.

5) Recovery from failure - Inquiry should be made on what would be the resultant effects on data and application in the case of disaster experienced by the system. Are there facilities for restoration and recovery? The time frame for the recovery should also be considered!

6) Investigative Ability - Are there effective monitoring facilities for easy detection and investigation of suspicious, inappropriate or illegal activity?

7) Long-Term Sustainability - Are there provisions for continuity of access to data in the case of vendors going out of business and in what format? This is to be confirmed to guarantee continuity of access to data

Cao [11], claims that the issue of data security is more critical in cloud computing than in traditional computing environment. A strategy not proposed by Gartner, in his work in [23], is the provision of facility for self-encryption by the consumers Irrespective of cloud service provider's security and encryption policies; data security and privacy can be assured when the consumers can self-encrypt their data and can only be accessed with predetermined decryption keys. The cloud security issues can be put into two categories, as presented follows:

\section{i) Security Challenges in Traditional System}

The security challenges in traditional systems are still present in cloud computing system but the advent of cloud computing system has given rise to a new wave of attack vectors. The new attack vectors make it possible and easier to carry out attack on services available in the cloud as a result of remote access to the facilities. The security and privacy application for authentication and authorization in enterprise environment may need to be improved to work with system in a cloud environment. Forensics monitoring and detection tasks might be difficult in a cloud computing environment because the investigators might not be able to have physical access to the hardware.

Cloud service providers are very important in the management of the cloud services and their involvement is also considered to be a big concern. They can help in the management of crises that rock cloud computing services because if the cloud services are disrupted, the effect is more severe than in the traditional system. For instance, "the recent disruption of the Amazon cloud service took down a number of websites including Reddit, Foursquare, and Quora due to a power outage. According to a recent report by the international working group on cloud computing resilience, it was estimated that the travel service provider, Amadeus loses $\$ 89,000$ per hour during any cloud computing outage, while PayPal loses around \$225,000 per hour". Furthermore, there are security issues inherent in virtual machine. "The hypervisor and virtual machines used by cloud providers may also have vulnerabilities exemplified. Such vulnerability represents even more serious problem in multi-tenant environments, where the compromise of a virtual machine can affect all users on the same physical server" [36].

Traditional security issues should, therefore, be reconsidered by cloud providers from different perspectives because of the peculiar nature of cloud computing environment.

\section{ii) Security Challenges in Cloud Computing}

In Cloud Computing, there are critical security concerns for privacy and user data integrity because the end-users make remote use of services available in the cloud and store data in the CSP's infrastructure. The end-users are interested in knowing how the data is stored, accessed and managed. They also want to be assured that vital information is not accessed by unauthorized users and used in unauthorized manners. Another area of concern is that the end-users only make use of services being provided by the cloud providers without knowing the exact location of the resources for such services, which is possibly in another domain. This poses a potential threat to end-users in the case of disputes or CSP moving out of business.

Rittinghouse and Ransome [37], argues that the legal frameworks of the country where cloud service providers are operating in addition to CSPs policies affect data stored by the providers.

To be able to use such services, users must agree to the "Terms of Service" which provide the CSP the right to disclose or use user data in compliance with provisions of the laws and law enforcement requests, for instance, "as noted in the recent Dropbox's Terms of Service. The European Union has issued Directive 95/46/EC to protect user privacy (European Commission, 2013). The directive prohibits transfers of personal data to countries which do not ensure an adequate level of protection" [37]. In European Union countries, it is allowed to transfer personal data of end-users outside EU countries if such is carried out after obtaining the consent of the owner of the data/information.

\section{E. Encryption Methods}

Sood in [38] describes as a process of transforming intelligible information into meaningless information. He 
emphasized that it is important to provide security for data in transit. The security of data being transmitted can be guaranteed via various cryptography schemes. In the case where the data gets into the hands of hacker, he would not be able to use the data until he decrypts it using appropriate decryption key or scheme.

Some of the encryption-decryption methods include private and public key encryption/decryption. "In a symmetric key (private key) encryption such as: DES, Triple DES, RC2, RC4, etc., the same key is used for encryption and decryption" [39]. Prior to the transfer of the data, the key is communicated by the sender to the receiver. The sender, therefore, sends the encrypted data and the receiver decrypts it using the same key by the sender as illustrated in Fig. 3 and 4. Prior to the transfer of the data, the key is communicated by the sender to the receiver. The sender, therefore, sends the encrypted data and the receiver decrypts it using the same key by the sender as illustrated in Fig. 3 and 4.

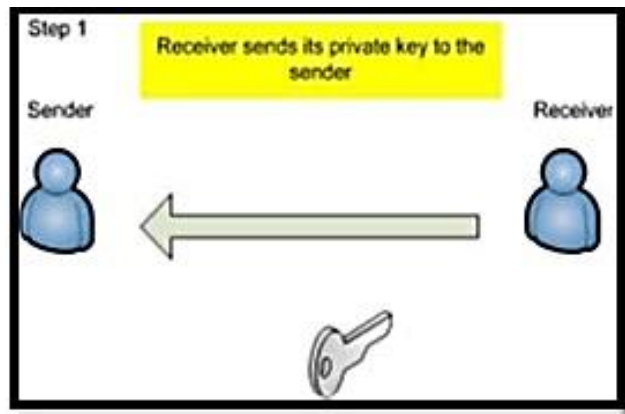

Fig. 3. Receiver send private key to the sender.

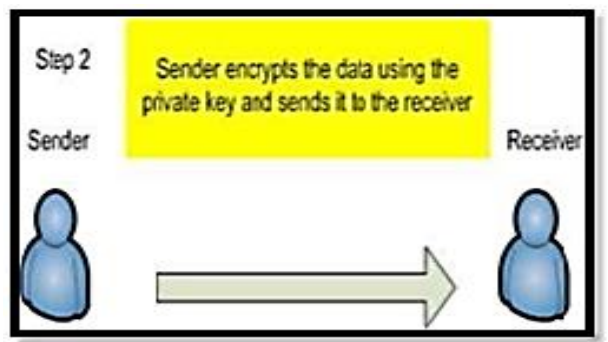

Fig. 4. Sender encrypts the data and send it to the receiver.

The method of encryption using public key might take a bit more processing time in when compared with private key encryption but in cases where security is the major concern instead of the speed of processing, public-key encryption offers more secured data transmission in relation to privatekey encryption. Secure Socket Layer (SSL) encryption also provides a key that permits only an authorized person to decode the sent information [38]. The SSL model implements this by using 128-bit SSL encryption to encrypt and index the as presented below:

$$
\text { 128-bits SSL (F, k2) } \rightarrow \text { F }
$$

As shown in the above, any key (k2) can be used to encrypt the file $\mathrm{F}$ and produces $\mathrm{F} 00$ (encrypted file) and this F0 0 can only be decrypted by the use of the same key k2 [38]. Figure 5 depicts how data is transferred from data owner to cloud [38].

A critical examination of 128-bit encryption using SSL will reveal that there are additional 288 bits of key length more than previous 40-bits encryption of SSL. The observed change signifies that there are additional 288 more combinations. This will make it tougher for hackers to crack the code. The range of the values is beyond the range of trillions which implies that 256-bit encryption using SSL is stronger. This provides for greater maintenance cost reduction. "The data is secured through SSL; AES based Cryptography, Server clustering and Server Load balancing" [38].

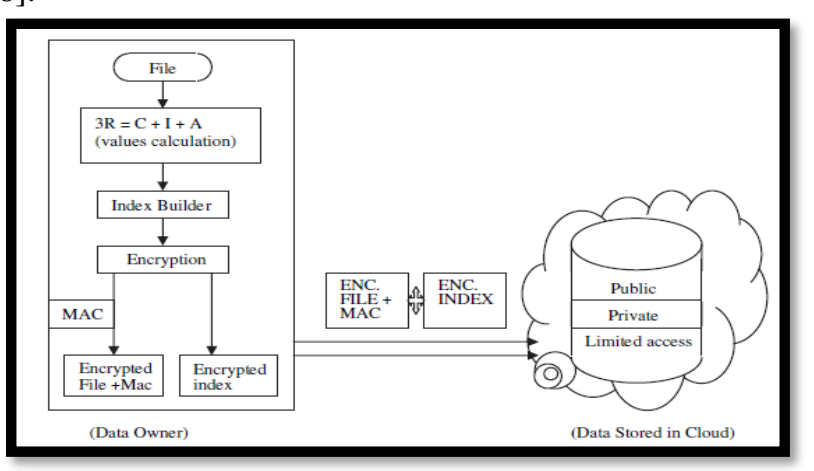

Fig. 5. Data transfer from owner to cloud [38].

\section{F. Legislations and Privacy Laws in Cloud Computing}

Cloud Computing may mean different things to different people but noticeably the privacy and security issues are different among consumers using public cloud application, medium-sized organisations using a computerized collection of business applications on a cloud platform, and government agencies with privately owned cloud for internal database sharing [40].

Different categories of users of cloud systems have their special package of benefits and risks associated with the usages. The tangible and intangible values remain constant as the users seek to protect data [41]. The value at risk for an individual ranges from loss of civil liberties to the contents of bank accounts [42]. Furthermore, the value for a business runs from core trade secrets to continuous business operations and maintaining public reputation. It is difficult to estimate some of these values and translate them into standard metrics of values [42]:

Main goals of information security are Confidentiality, Integrity, and Availability as explained below with regards to the concept of cloud computing:

- Confidentiality: This can be understood as keeping data as private as possible. Privacy is very important as data departs the borders of an enterprise [41]. It is not enough to only protect the internal secrets and sensitive personal but also the fundamental metadata and transactional data are not supposed to be leaked about individuals and organisations. "Confidentiality is supported by technical tools such as encryption and access control, as well as legal protections"

- Integrity: This can be defined as the degree of correctness attached to data saved in the cloud and how it is protected against accidental or intentional alteration without authorization [41]. It covers, also, the problems of effective synchronization of multiple databases. Integrity has been supported by effective audited code, properly designed distributed systems and strong access control methods.

- Availability: This means being able to use the system as anticipated when the needs arise. Cloud technologies can increase the availability through widespread internetenabled provision and the customers are dependent on the timely and robust provision of needed resources. Service 
providers' capacity building, effective architecture and welldrafted contracts and agreement terms and conditions can support availability [41].

Most scholars consider privacy as a concern in Cloud computing [43]. Different countries have different Privacy laws and legislations that govern data storage in the cloud. For example, The European Union Data Protection Directives (DPD) provisions affect personal data that are processed in the cloud. The provisions also cover the activities of those providing cloud services being used to process users data. Moreover, DPD has been identifies not to be strictly privacy law [43]. In UK and Europe, Data Privacy laws prevent the movement of data outside the jurisdiction which can be the direct opposite of the multitenancy concept of cloud computing [44].

In America, privacy law associated with cloud platforms is put on a lower scale than that those associated with personal computers and hand-held mobile gadgets [45]. It has been accorded lower protection by allowing law enforcement agencies access to any information without fourth $\left(4^{\text {th }}\right)$ amendment warrant prerequisites. This has created much uncertainties regarding the powers United State government and other sovereign powers over data stored in the cloud [41].

For the purpose of national security, some countries prefer storing and accessing their data within their territory and hold onto the view that the only system they trusted is one operated within their own jurisdictions [41]. For example, In Europe, there is well enforced data protection directive that prevents data movement from the European Union countries to countries with privacy and confidentiality rules. This practice of transfer of data has been considered inappropriate. The DPD employs binary principle of "all or nothing" to data. If information is considered "personal data", all rule applicable to data protection are applied (not just some rules) and otherwise, DPD rules do not apply to data [43].

\section{ANALYSIS OF THE EXISTING GUIDES}

There are several bodies who have been publishing material relating to cloud computing security, including, but not limited to: CSA, NIST, CPNI (Centre for the Protection of National Infrastructure from UK government) and ISACA (the Information Systems Audit and Control Association). After thorough evaluation of the available published documents, it is evident that there is no one guide suitable for the purpose of this research. Therefore, it is necessary to focus on the first three bodies whose works provide a comprehensive overview of issues and solutions relating to the Cloud; thus, enabling a broad understanding of the current status of cloud security. These documents consist of cloud computing security guidelines which have been reviewed to produce suitable guides and recommendations on how to secure data stored in the cloud.

\section{CONCLUSION AND RECOMMENDATIONS}

Cloud computing has been identified as a computing paradigm that can access, conveniently, a dynamic and configurable public set of computing resources, including data storage, among others. These cloud resources are made available rapidly on-demand with least providers' intervention. However, the predominance of cloud computing is being challenged by a number of security issues and threats to a great extent. The research has explored and analyzed three main existing cloud security guidelines to identify a list of best practices to promote the security of data and other services in the cloud in order to guide cloud service providers and end-users. This suggests that selection of appropriate cloud service providers with secured infrastructure, compliance with related laws and policies as well as adequate knowledge of end-users on available security apparatus are essential for adequate privacy and security of data in the cloud.

\section{REFERENCES}

[1] L.M. Vaquero, L. Rodero-Merino, J. Caceres and M. Lindner, "A break in the clouds: Towards a cloud definition," ACM SIGCOMM Comput. Commun. Rev., 39: 50-55, 2009.

[2] Computer Infographics (May 2013). "Infographic Zone: The No. 1 source of data visualization," [Online]. Available: http://www.infographicszone.com/computers/top-5-cloudcomputing-infographics

[3] I. P. Sokolov, "Cloud Computing: Overview, Concepts and Business Development Scenerio," Thesis, Vienna University of Economics and Business:, p. 9, 2009.

[4] J. Mckendric (2012), "Forbes", [Online]. Available: http://www.forbes.com/sites/joemckendrick/2012/02/22/6-shiningexamples-of-cloud-computing-in-action/

[5] M. Armburst, A. Fox, R. Griffith, A. D. Joseph, R. Katz, A. Konwinski, G. Lee, D. Pattersson, A. Rabkin, I. Stoica and M Zaharia, "Above the Clouds: A Berkeley View of Cloud Computing," Berkeley: EECS Department, University of Califonia, 2009.

[6] ORACLE (2012), "Oracle's Cloud Solutions for Public Sector," [Online]. Available: http://www.oracle.com/us/industries/publicsector/cloud-solutions-public-sector-wp-323002.pdf

[7] D. Farber. (2006), "Oracle's Ellison nails cloud computing," [Online]. Available: http://news.cnet.com/8301-13953_3-1005218880.html.

[8] Intel IT Centre. (2012), "Planning guide cloud security," pp. 5.

[9] Z. Whittaker. (2012), "Amazon Web Services suffers partial outage," [Online]. Available: http://www.zdnet.com/blog/btl/amazon-webservices-suffers-partial-outage/79981.

[10] M. J. Schwartz. (2011), “6 Worst Data Breaches of 2011,” [Online]. Available:

http://www.informationweek.com/news/security/attacks/232301079.

[11] N. Cao, (2012) "Secure and reliable data outsourcing in Cloud computing," Worcester Polytechnic Institute: S.N, 2012.

[12] IDC. (2011). "Cloud will have dramatic impact on IT organisations, vendors and enterprises," [Online]. Available: Accessed $13^{\text {th }}$ March 2010 http://www.idc.com/getdoc.jsp?containerId=prUS22903111.

[13] G. Reese, "Cloud Application Architectures: Building Applications and Infrastructure in the Cloud,". S.L.:O'Reilly, (2009).

[14] R. Jacob, "Security in the Cloud: The threat of coexist with an unknown tenant on a public environment", Royal Holloway, University of London: s.n., 2012.

[15] CSIS. (2009), "Threats Posed by the Internet," [Online]. Available: http://csis.org/files/media/csis/pubs/081028_threats_working_group. pdf.

[16] Cloud Security Alliance. (2011), "Encription and key management,", [Online].

Available:https://wiki.cloudsecurityalliance.org/guidance/index.php/ Encryption_and_Key_Management.

[17] NIST. (2009), “The NIST definition of cloud computing,". [Online]. Available: http://csrc.nist.gov/publications/nistpubs/800-145/SP800145.pdf.

[18] T. Grance. (2011). "The NIST Definition of Cloud Computing" [Online]. Available at: http://www.nist.gov/itl/cloud/upload/clouddef-v15.pdf.

[19] CSA. (2010). Encription and key management. [Online] Available at: https://wiki.cloudsecurityalliance.org/guidance/index.php/Encryption and_Key_Management.

[20] CSA, "Security Guidance for Critical Area of Focus in Cloud computing," Cloud Security Alliance, V2.1.p. 33, (2009). 
[21] F. Jericho. (2009). "Cloud Cube Model". [Online]. Available: http://www.opengroup.org/jericho/cloud_cube_model_v1.0.pdf.

[22] ENISA. (2009). "Cloud computing: Benefits, risks and recommendations for information security," [Online]. Available: http://www.enisa.europa.eu/act/rm/files/deliverables/cloudcomputing-risk-assessment.

[23] Gartner. (2011). "CIO Agenda Findings," [Online]. Available: http://www.gartner.com/technology/cio/cioagenda_findings.jsp.

[24] T. J. Velte, R. Elsenpeter. (2010). "Cloud computing: A practical Approach," s.l.:McGraw - Hill.

[25] R. L. Krutz et al, "Cloud Security: A Comprehensive Guide To Secure Cloud Computing," 2010.

[26] Global Knowledge. (2011). "10 security concerns for cloud computing,". [Online]. Available at: http://viewer.media.bitpipe.com/1078177630_947/1268847180_5/W P_VI_10SecurityConcernsCloudComputing.pdf.

[27] R. Murch (2004), “Autonomic Computing,”. s.l.:IBM press, pp. 336, 2004

[28] M. Rouse. (2006), "Autonomic Computing," [Online]. Available at: http://searchcio-midmarket.techtarget.com/definition/autonomiccomputing.

[29] P. Horn. (2001), "Autonomic Computing: IBM perspective on the state of information technology," [Online]. Available at: http://www.research.ibm.com/autonomic.

[30] J. Kephart, "The Vision of Autonomic Computing," IEEE Comput 2003; vol. 36, pp. 41 - 50. 2003

[31] IBM, "Autonomic Computing: IBM perspective on the state of information technology," IBM J., 2003.

[32] R. Buyya, C. S. Yeo, and S. Venugopal (2008), "Market-oriented cloud computing: Vision, Hype, and reality for delivering IT services as Computing Utilities,", Keynote Paper, China, Dalian, 2008.

[33] D. Neumann, M. Baker, J. Altmann and O.F. Rana, (2010) "Economic Models and Algorithm for Distributed Systems," Birkauser, Berlin, 2010.

[34] Gridpedia. (n.d). "The European Grid Marketplace," [Online]. Available at: http://www.gridipedia.eu/sla-article.html.

[35] F. Liu, J. Tong, J. Mao, R. Bohn, J. Messina, L. Badger and D. Leaf, "NIST Cloud computing reference architecture; Recommendations of the National Institute of Standards and Technology," Gaithersburg: NIST, 2011.

[36] O. Florence. (2012). "The Effects of The Amazon Web Services Outages," [Online]. Available: http://www.cloudtweaks.com/2012/06/the-effects-of-the-amazonweb-services-outages/.

[37] J. W. Rittinghouse and J. F. Ransome, "Cloud Computing: Implementation, Management, and Security," Auerbach Publications, New York, 2009.

[38] S. K. Sood. "A combined approach to ensure data security in cloud computing,", Gurdaspur, India: Department of Computer Science and Engineering, GNDU, 2012.

[39] T. Dierks and C. Allen, "The TLS Protocol, Certicom: Network Working Group," The Internet Society, 1999.

[40] A. Whitten, "Consumer Online Privacy: Statement at hearing of Senate Committee on Commerce, Science, and Transportation", July 272010 .

[41] A. Friedman, A. Allan, and M. D. M. West, "Privacy and Security in Cloud Computing," Issues of Technology Innovation, pp. 1, 2010.

[42] B. Lev, "Remarks on the Measurement, Valuation, and Reporting of Intangible Assets," FRBNY Economic Policy Review. 2003.

[43] K. Hon. (2011). "Data protection, the law and you," [Online]. Available:http://blogs.computerworlduk.com/cloudvision/2011/04/data-protection-the-law-and-you-1/index.htm.

[44] D. Preez. (2011). "UK census contractor says data privacy laws will have to change," [Online]. Available: http://www.computing.co.uk/ctg/news/2029852/uk-censuscontractor-privacy-laws-change.

[45] A. Oza, (2008). Amend the ECPA: Fourth Amendment Protection Erodes as E-Mails Get Dusty. BUL Rev., 88

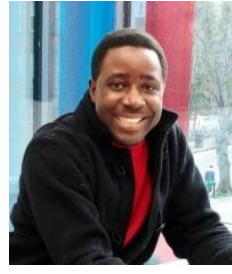

A. Abubakar received his BSc. in Computer Science from Usmanu Danfodiyo University, Sokoto, Nigeria, in 2007, and his MSc in Software Engineering from University of Southampton, UK, in 2012.

Abdullahi is an active member of several professional bodies including Institute of Electrica and Electronics Engineers (IEEE), Nigerian Computer Society (NCS), British Computer Society (BCS), International Association of Engineers (IAENG), Institute for Systems and Technologies of Information, Control and Communication (INSTICC). He is currently a lecturer at the Department of Computer Science, Waziri Umaru Federal polytechnic, Birnin Kebbi, Nigeria.

His research interests include data science, cloud computing, machine learning, big data (parallel and distributed processing) and anomaly detection. He is married and has children.

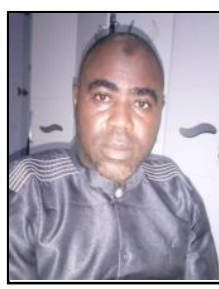

A. I. Babate holds a Bachelor of Technology (B.Tech.) Degree in Computer Science from Abubakar Tafawa Balewa University (ATBU) Bauchi, Master of Computer System Security (MSc.) from University of South Wales, United Kingdom.

$\mathrm{He}$ is an NCS registered Member Computer Society of Nigeria. Member Computer Professionals of Nigeria (CPN). Member International Institutes of Research Engineers and Doctors (theIRED). Member Teachers' Registration Council of Nigeria (TCRN).

$\mathrm{He}$ is currently a lecturer at the Department of Computer Science, Federal College of Education (Tech.) Potiskum Yobe State, Nigeria. He is Married and has children.

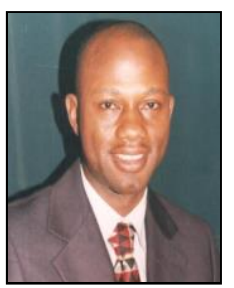

A. Ishola holds a Bachelor's Degree of Computer Science (B.Sc), from Usmanu Danfodiyo University, Sokoto. Masters of Science Degree in Computer Science (M.Sc) from Kebbi State University of Science and Technology Aleiro, Kebbi State.

$\mathrm{He}$ is currently a lecturer at Federal Polytechnic Birnin Kebbi, Kebbi State, Nigeria, in the Department of Computer Science.

$\mathrm{He}$ is a registered member of Nigerian Computer Society (NCS) and International Association of Engineers (IAENG). $\mathrm{He}$ is married and blessed with children.

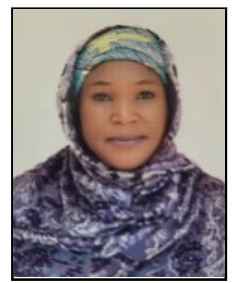

A. M. Sani hold Bachelor of Technology (B.Tech) Degree in Computer Science Education from Federa University of Technology (FUT) Minna, Niger State, Nigeria.

She is currently a lecturer at the Department of Computer Science Educational, College of Technical and Vocational Education (CTVE), Kaduna Polytechnic, Kaduna, Nigeria

She is married and blessed with children. 